\title{
Estudio de los enfoques de aprendizaje en estudiantes de Magisterio y Psicopedagogía
}

\author{
Ana Belén García Berbén ${ }^{1}$ \\ Departamento de Psicología Evolutiva y de la Educación, \\ Universidad de Almería
}

\section{España}

berben@ual.es

\footnotetext{
${ }^{1}$ Becaria FPI. Esta investigación fue realizada gracias a la ayuda del Proyecto I+ D ref. BSO2003-06493. Ministerio de Educación y Ciencia. España.
} 


\section{Resumen}

Introducción. La nueva cultura de la calidad de las universidades y las reformas en los planes de estudios provocan la necesidad de conocer la realidad educativa, especialmente los procesos de estudio que los estudiantes universitarios desempeñan en su aprendizaje académico. Este trabajo pretende analizar cuáles son las características del aprendizaje de los estudiantes y si existen diferencias en sus procesos de estudio en relación a las características individuales de edad y género.

Método. En el estudio participan 215 alumnos y alumnas de la Universidad de Granada. Se utilizó el revised two-factor Study Process Questionnaire (Biggs, Kember y Leung, 2001), para medir el enfoque de aprendizaje que éstos adoptan en sus tareas académicas.

Resultados. Se obtiene un porcentaje pequeño y similar de alumnado que adopta un enfoque predominantemente profundo o predominantemente superficial. La mayoría del alumnado se muestra más motivado intrísecamente; sin embargo, utiliza estrategias de aprendizaje tanto memorísticas como comprensivas. Tanto el género como la edad influyen en los enfoques de aprendizaje adoptados por los participantes del estudio. Las alumnas utilizan significativamente más un enfoque profundo que los alumnos, y el alumnado de 26 o más edad estudia significativamente más con un enfoque profundo que sus compañeros de menor edad.

Discusión. Las características encontradas en los procesos de estudios de los universitarios aportan claves importantes para acomodar los nuevos créditos ECTS a las peculiaridades de la realidad del aprendizaje académico de estos estudiantes. Los procesos de estudio centrado exclusivamente en el material obligatorio, junto a las diferencias encontradas según la edad y el sexo, sugieren que es necesario seguir investigando en esta línea.

Palabras Clave: diferencias individuales, enfoques de aprendizaje, aprendizaje académico, educación superior. 


\section{Introducción}

El aprendizaje promovido en las universidades es un tópico de estudio menos investigado que en etapas educativas inferiores. Actualmente, la investigación del aprendizaje se ha interesado en la educación superior por diversas razones, entre las que habría que destacar, por una parte, el fracaso académico y abandono de los estudios (García, Salvador y Zubieta, 1991) y, por otra parte, el interés de las leyes y reformas educativas de ofrecer una universidad de calidad. El Espacio Europeo de Educación Superior (EEES) pretende entre otros objetivos, promover el "aprender a aprender" del estudiante y ofrecerle la posibilidad de ser un aprendiz experto a lo largo de su vida.

En la perspectiva cognitiva coexisten diferentes líneas de investigación interesadas en el estudio del aprendizaje académico. Una de estas líneas es la fenomenográfica. El marco teórico de la fenomenografía defiende el concepto de "enfoques de aprendizaje" (approach learning) para referirse al modo en que los estudiantes se enfrentan a la tarea. La fenomenografía es entendida como una perspectiva de segundo orden (Marton, 1981), ya que se interesa por cómo la realidad es percibida por la persona que la vive; en este caso, cómo el alumnado percibe su aprendizaje. Es desarrollada principalmente por investigadores europeos y australianos, aunque comienza a extenderse a otros países asiáticos, como China.

Según los investigadores de esta línea, el aprendizaje académico se relaciona más con el contexto de aprendizaje que con las características personales del estudiante (Biggs, 1993). Esta peculiaridad permite la mejora de los enfoques cuando se modifican las características del contexto. La posibilidad de mejora convierte a los enfoques en un referente importante para la investigación e intervención en el aprendizaje académico. Algunos grupos de investigación en este marco de estudio (Barca, Porto y Santorum, 1997; Biggs, 1993, 2001b; Cano y Justicia 1994; Hernández Pina,1993, 1997;) son: (a) el grupo de Marton y Säljö; estos autores fueron los primeros en estudiar los enfoques de aprendizaje; (b) el grupo de Noel Entwistle, que se encuentra influenciado por los estilos de aprendizaje de Pask y las orientaciones hacia la educación de Taylor. Entwistle añade un trecer enfoque u orientación: el enfoque estratégico (strategic approach); y (c) el grupo de Biggs. 
Las investigaciones de Biggs suelen ser de corte cuantitativo, ya que la recogida de datos la realizan con cuestionarios construidos por ellos mismos. Biggs concibe los enfoques como un compuesto de motivación y estrategias de aprendizaje y añade un tercer enfoque, el enfoque de logro (achieving approach). Los objetivos de este trabajo y las pretensiones con las que se inicia el estudio se relacionan más con el concepto de enfoque propuesto por Biggs.

Desde la teoría de los enfoques de aprendizaje del estudiante (Students Approach to Learning, SAL), Biggs propone tres enfoques diferentes (Biggs, 2001a, 2001b).

- Enfoque Superficial (surface approach), se compone de una motivación extrínseca e instrumental. La intención es satisfacer los requisitos de la tarea con el mínimo esfuerzo, evitando el fracaso. Utiliza como estrategias la memorización y la reproducción mecánica, limitándose a lo esencial, centrándose en aspectos concretos y literales, y evitando establecer relaciones entre los temas. Existe una preocupación por el tiempo invertido en la tarea.

- Enfoque Profundo (deep approach), se caracteriza por una motivación intrínseca y una preocupación por comprender, adoptando de este modo estrategias que llevan al significado inherente de la tarea. El alumnado que adopta este enfoque intenta relacionar los contenidos con contextos personales significativos o con conocimientos previos y encuentra el aprendizaje emocionalmente satisfactorio.

- Enfoque de logro (achieving approach), en este caso el objetivo es manifestar la propia competencia con respecto a los compañeros, intentando obtener las máximas calificaciones. El alumnado utiliza como estrategia la optimización del coste-eficacia del tiempo y el esfuerzo. Considera importante la autodisciplina, el orden y la sistematización, la planificación y la distribución del tiempo.

Biggs, Kember y Leung (2001) han desarrollado una nueva versión tras la revisión del cuestionario utilizado hasta el momento para evaluar los procesos de estudios de los universitarios (Study Process Questionnaire, SPQ). La nueva versión reduce los ítems de 43 a 20 y las subescalas, de seis a cuatro. El cuestionario reducido se denomina Revised two-factor Study 
Process Questionnaire (R-SPQ-2F), debido a que su objetivo es medir sólo dos factores o enfoques (profundo-superficial).

El estudio de la unidimensionalidad y fiabilidad del R-SPQ-2F muestra un coeficiente alpha de Cronbach de 0,73 para el enfoque profundo y 0,64 para el enfoque superficial (Biggs y otros, 2001). En el ámbito internacional el R-SPQ-2F ha sido utilizado y analizado por diversos autores en diferentes culturas y contextos universitarios (Harris, Wickline, y Iliescu, 2004; Leung y Chan, 2001; Pilcher, 2002); sin embargo, este instrumento ha sido poco utilizado en nuestro país (Hernández Pina, García, Martínez, Hervás y Maquilón, 2002).

Los estudios desarrollados tradicionalmente desde la teoría de los enfoques de aprendizaje de los estudiantes (SAL) se centran principalmente en la relación existente con variables y características del contexto educativo, tales como métodos de evaluación, métodos de enseñanza, características del profesorado y rendimiento académico. Estos estudios muestran relaciones e influencias de dichas variables sobre los enfoques. En este sentido, los autores defienden una relación positiva entre los métodos de enseñanza centrados en la transmisión del contenido y el enfoque superficial, así como entre métodos de enseñanza centrados en el alumnado y el enfoque profundo (Buendía y Olmedo, 2000; Entwistle y Tait, 1990; Kember y Gow, 1994; Meyer, Dune y Richardson, 1994; Olmedo, 2001; Trigwell, Prosser y Waterhouse, 1999).

Otras investigaciones están interesadas en la influencia de las diferencias individuales de los universitarios, tales como la edad y el género (Buendía y Olmedo, 2002; Dune y Richardson, 1994; Hernández Pina y otros, 2002; Sadler-Smith y Tsang, 1998; Zhang, 2000).

Los resultados y conclusiones sobre las diferencias de género son variados, relacionando de forma diferente el tipo de enfoque con hombres y mujeres. Hernández Pina y otros (2002) obtienen medias muy similares entre hombres y mujeres en el enfoque superficial y profundo. Igualmente, Buendía y Olmedo (2002) no encuentran diferencias significativas en los enfoques adoptados según el género, pero sí se observan diferencias al analizar más detenidamente los ítems del SPQ. Por otra parte, Cano (2000) utiliza el Approaches to Studying Inventory de Entwistle (Entwistle y Ramsden, 1983), para estudiar la influencia del género en los enfoques de universitarios. Este autor concluye que las alumnas superan a los alumnos en enfoque superficial y estratégico. 
En investigaciones internacionales, Sadler-Smith y Tsang (1998), en su estudio sobre el aprendizaje de universitarios de Hong-Kong, concluyen que los hombres están motivados más profundamente que las mujeres. En la misma línea, Zhang (2000), al estudiar los enfoques de aprendizaje en tres muestras distintas (Estados Unidos, China y Hong-Kong), obtiene que los alumnos chinos están motivados más profundamente que las alumnas chinas.

Las diferencias de género en el aprendizaje sí han sido más estudiadas desde perspectivas diferentes a la SAL. En algunos de estos estudios (Cano, 2000; Fuente, Justicia, Archilla y Soto, 1994; Martín y Camarero, 2001) se han investigado las diferencias de estrategias y estilos de aprendizaje según el género y la titulación universitaria. Los resultados de sus investigaciones muestran que sí hay diferencias en las estrategias y estilos de aprendizaje entre alumnas y alumnos, así como la influencia del tipo de titulación en estas diferencias. De entre las diferencias encontradas por los autores, en este estudio interesan los resultados obtenidos por Martín y Camarero (2001). Estos analizan distintas especialidades de los Títulos de Magisterio y obtienen diferencias significativas según el género; sin embargo, no se encuentran diferencias entre las distintas especialidades, ni entre los tres cursos.

El estudio de la influencia que ejerce la edad sobre los enfoques de aprendizaje está menos desarrollado que el género. Se encuentra poca fundamentación teórica sobre la evolución del aprendizaje y los procesos de estudio de los universitarios. En general, las investigaciones apenas justifican las diferencias de edad, dificultando la posibilidad de establecer criterios que fundamenten suficientemente los intervalos de edad. Los resultados de algunas de estas investigaciones muestran que el alumnado de mayor edad utiliza más un enfoque profundo, mientras que los estudiantes de menor edad adoptan más el enfoque superficial (Hernández Pina y otros, 2002; Richardson, 1995; Sadler-Smith, 1996). Por otro lado, Zhang (2000) concluye que los participantes de mayor edad de Estados Unidos y China adoptaron significativamente menos el enfoque de logro.

Tras la revisión realizada, en este trabajo se plantean dos objetivos. Por un lado, se desea conocer qué tipo de enfoque de aprendizaje posee el alumnado universitario estudiado. Por otro lado, analizar la influencia de diferentes variables personales (sexo y la edad) en los enfoques de aprendizaje de los universitarios. 


\section{Método}

\section{Participantes}

La muestra está compuesta por 215 estudiantes de primer curso de la Facultad de Ciencias de la Educación de la Universidad de Granada. El 53,5\% de los estudiantes se encuentra matriculado en diferentes especialidades de la Titulación de Magisterio, y el 46,5\% en Psicopedagogía. El 82,8\% de la muestra son mujeres y 17,2\% hombres. El 21,4\% del alumnado tiene 19 ó menos años, el 31,1\% tiene entre 20 y 22 años, el 28,8\% entre 23 y 25 años, y el $11,6 \%$ tiene 26 años ó más.

\section{Variables e instrumentos}

El modelo de Biggs estudia las estrategias utilizadas por el alumnado en su aprendizaje y la motivación de este alumnado para aprender. Estas estrategias y motivaciones corresponden a las variables estudiadas siendo denominadas y definidas como sigue (Biggs, 2001a, 2001b):

- Estrategia profunda: se trata de las estrategias necesarias en la comprensión de la tarea, de su significado, por ejemplo el uso de analogías, metáforas.

- Estrategia superficial: la estrategia que se utiliza para el aprendizaje es la reproducción del material a través de la repetición.

- Motivación profunda: el interés o motivación es intrínseco a la tarea, el alumnado con esta motivación tiene la intención de conocer el significado y los principios sin considerar el esfuerzo necesario.

- Motivación superficial: esta motivación es extrínseca al propósito de la tarea, el alumnado que la posee tiene la intención de aprender para evitar el fracaso con el menor esfuerzo posible.

Las variables de estudio se agrupan en un tipo de enfoque que puede ser profundo, cuando se suman los valores de estrategia y motivación profunda, o superficial, cuando se suman estrategia y motivación superficial (Biggs y otros, 2001; Fox, McManus y Winder, 2001; Hernández Pina y otros, 2002; Leung y Chan, 2001; Pilcher, 2002). 
Las variables estudiadas se miden a través del The revised two-factor study process questionnaire (R-SPQ-2F), construido por Biggs y otros (2001), que ha sido traducido y adaptado al castellano por De la Fuente y Martínez (2003) como "Cuestionario de Procesos de Estudios Revisado".

Los valores que hemos obtenido del análisis de la fiabilidad son similares a los de otros autores (Biggs y otros, 2001; Leung y Chan, 2001). Los datos de este estudio muestran un coeficiente alpha de Cronbach de 0,78 para el enfoque profundo y 0,76 para el enfoque superficial. Por otra parte, se ha estudiado la congruencia mediante la prueba de Correlación de Pearson. Los resultados muestran que la subescala de motivación profunda es congruente con la de estrategia profunda $(\mathrm{r}=0,654 ; \mathrm{p}<0,01)$, e incogruente con los dos componentes del enfoque superficial: motivación $(r=-0,396 ; p<0,01)$ y estrategia $(r=-0,362 ; p<0,01)$. A su vez, la estrategia profunda es incongruente con la estrategia superficial $(r=-0,448 ; p<0,01)$ y con la motivación superficial $(r=-0,464 ; \mathrm{p}<0,01)$. Por último, se obtiene que los componentes del enfoque superficial son congruentes entre sí $(r=0,662 ; p<0,01)$.

\section{Análisis Estadístico}

Para responder al primer objetivo se estudian la frecuencia y estadísticos descriptivos (media y desviación típica) de los enfoques de aprendizaje que adopta el alumnado. Para el segundo de los objetivos, se utiliza la prueba $T$-student para analizar la influencia del sexo y el $A N O V A$ de un factor para las diferencias según la edad. El paquete estadístico utilizado para estos análisis es el SPSS 10.0.

\section{Resultados}

\section{Enfoques adoptados por los estudiantes}

Las respuestas de los participantes ofrecen una media mayor en el enfoque profundo $(X=31.54)$ que en el superficial $(X=26.55)$. Del mismo modo, las escalas que obtienen las medias más altas son las escalas de motivación profunda $(\mathrm{X}=16.27)$ y estrategia profunda $(X=15.28)$, seguidas de la estrategia superficial $(X=15.10)$ y la motivación superficial $(\mathrm{X}=11.45)$. Sin embargo, estos datos no pueden demostrar por sí sólos que los participantes adopten en mayor medida un enfoque que otro. 
Para conocer qué tipo de enfoque adoptan los participantes se consideran los criterios establecidos por Biggs (citado en Barca, Porto y Santorum, 1997). Utilizando estos criterios se pueden obtener dos categorías de enfoque profundo (exclusivo o predominante) y dos de enfoque superficial (exclusivo o predominante).

El porcentaje del alumnado que estudia con un enfoque exclusivo (ya sea superficial $=3,7 \%$ o profundo $=3,3 \%$ ), es menor que el que utiliza un enfoque predominante (superficial $=19,5 \%$ o profundo $=18,6 \%)$. Sin embargo, la mayoría de los participantes $(54,9 \%)$ no cumplen los criterios predeterminados, por ello se les denomina alumnado "no catalogado". Para conocer las características de este último grupo, se analizan sus respuestas más detalladamente. En la Tabla 1 se presentan las medias de las puntuaciones en cada uno de los ítems del R-SPQ-2F del alumnado "no catalogado".

Tabla 1. Medias de las puntuaciones en los ítems del R-SPQ-2F de alumnado no catalogado

\begin{tabular}{|c|c|c|c|c|}
\hline \multirow[t]{2}{*}{ Ítem } & \multicolumn{2}{|c|}{ Enfoque Profundo } & \multicolumn{2}{|c|}{ Enfoque Superficial } \\
\hline & Motivación & Estrategia & Motivación & Estrategia \\
\hline 1. & 3,36 & & & \\
\hline 2. & & 3,4 & & \\
\hline 3. & & & 2,38 & \\
\hline 4. & & & & 3,78 \\
\hline 5. & 2,97 & & & \\
\hline 6. & & 2,88 & & \\
\hline 7. & & & 1,96 & \\
\hline 8. & & & & 2,57 \\
\hline 9. & 3,45 & & & \\
\hline 10. & & 3,69 & & \\
\hline 11. & & & 2,44 & \\
\hline 12. & & & & 2,75 \\
\hline 13. & 3,18 & & & \\
\hline 14. & & 1,99 & & \\
\hline 15. & & & 1,83 & \\
\hline 16. & & & & 3,2 \\
\hline 17. & 3,13 & & & \\
\hline 18. & & 3,12 & & \\
\hline 19. & & & 2,5 & \\
\hline 20. & & & & 2,53 \\
\hline
\end{tabular}

Al analizar las respuestas del alumnado "no catalogado", se observa que las dos subescalas del enfoque profundo (estrategia y motivación) muestran medias similares entre sí, mientras que entre las dos subescalas del enfoque superficial (estrategia y motivación) estos valores son más distantes. El porcentaje de alumnos/as que dicen poseer una motivación pro- 
funda es similar a los que dicen utilizar estrategias profundas. Por otro lado, se encuentran más estudiantes que dicen usar estrategias superficiales, que quienes dicen poseer una motivación superficial (Tabla 1).

Los ítems en los que el alumnado arroja medias más altas son los referidos a:

(4) Estudio en serio sólo lo que se trata en la clase o lo que viene en el programa de estudios. (I only study seriously what's given out in class or in the course outline)

(9) A veces siento que estudiar temas académicos puede ser tan emocionante como una buena novela o película. (I find that studying academic topics can at times be as exciting as a good novel or movie)

(10) Me hago pruebas sobre los temas importantes hasta que los comprendo por completo. (I test myself on important topics until I understand them completely)

Mientras que los ítems en los que las medias son más bajas son:

(7) No encuentro interesante mi curso/asignatura, así que le dedico un mínimo de trabajo. ( do not find my course very interesting so I keep my work to the minimum)

(14) Paso mucho de mi tiempo libre averiguando más sobre los temas interesantes discutidos en las distintas clases. (I spend a lot of my free time finding out more about interesting topics which have been discussed in different classes).

(15) Observo que no es conveniente estudiar los temas a fondo. Produce confusión y se pierde tiempo, cuando sólo hace falta conocer los temas por encima. (I find it is not helpful to study topics in depth. It confuses and wastes time, when all you need is a passing acquaintance with topics).

Género y enfoques de aprendizaje

El género influye significativamente en el enfoque adoptado por el alumnado. Las alumnas utilizan significativamente más las estrategias profundas y poseen mayor motivación profunda que los alumnos. En cambio, los alumnos poseen una motivación superficial significativamente superior a las alumnas y utilizan más las estrategias superficiales que ellas. Estos resultados se reflejan inevitablemente en los enfoques adoptados por ambos. De este modo, las alumnas adoptan más el enfoque profundo y los alumnos adoptan más un enfoque superficial (Tabla 2). 
Tabla 2. Diferencias de medias en función del sexo en las escalas y enfoques adoptados por el alumnado $(\mathbf{p}<0,01: * * ; p<0,05: *)$.

\begin{tabular}{lccccc}
\hline \multirow{2}{*}{ Escalas y Enfoques } & \multicolumn{2}{c}{ Alumnos } & \multicolumn{2}{c}{ Alumnas } & \multirow{2}{*}{$\mathbf{\text { t }}$} \\
\cline { 2 - 5 } & $\mathbf{X}$ & $\mathbf{d t}$ & $\mathbf{X}$ & $\mathbf{d t}$ & \\
\hline Motivación profunda & 15,30 & 3,25 & 16,47 & 3,01 & $-2,01^{*}$ \\
Estrategia profunda & 13,89 & 3,18 & 15,57 & 3,11 & $-2,92^{* *}$ \\
Motivación superficial & 13,95 & 3,33 & 10,93 & 3,37 & $4,9^{* *}$ \\
Estrategia superficial & 16,62 & 3,03 & 14,79 & 3,35 & $3,28^{* *}$ \\
\hline Enfoque profundo & 29,19 & 5,74 & 32,03 & 5,57 & $-2,75^{* *}$ \\
Enfoque superficial & 30,57 & 5,52 & 25,72 & 6,15 & $4,76^{* *}$ \\
\hline
\end{tabular}

\section{Edad del alumnado y sus enfoques de aprendizaje}

Los resultados muestran que la edad del alumnado influye significativamente en sus enfoques de aprendizaje. Realizado el análisis post-hoc, se obtiene que el alumnado de 26 años en adelante es significativamente diferente a sus compañeros de menor edad en todos los componentes, exceptuando la motivación profunda. El grupo de edad de más de 26 años adopta significativamente más el enfoque profundo que los grupos de 20-22 y 23-25 años. A su vez, el grupo de mayor edad adopta significativamente un enfoque menos superficial que el resto (Tabla 3).

El análisis de las subescalas muestra que el alumnado de más de 26 años aborda las tareas con estrategias significativamente más profundas que el resto de compañeros y posee una motivación más profunda, aunque ésta no es significativa. Este alumnado de mayor edad también posee significativamente menos motivación superficial y utilizan menos estrategias superficiales que los alumnos de edades comprendidas entre 20-22 y 23-25 años.

Tabla 3. Escalas y enfoques adoptados por el alumnado según la edad

\begin{tabular}{|c|c|c|c|c|c|}
\hline \multirow[t]{2}{*}{ Escalas y Enfoques } & -19 & $20-22$ & $23-25$ & +26 & \multirow[t]{2}{*}{$\mathbf{F}$} \\
\hline & $X(d t)$ & $X(d t)$ & $X(d t)$ & $X(d t)$ & \\
\hline Motivación profunda & $15,91(2,55)$ & $16,01(3,06)$ & $16,13(2,94)$ & $18,08(3,79)$ & $3,43^{*}$ \\
\hline Estrategia profunda & $15,30(3,22)$ & $14,74(2,68)$ & $15,05(3,18)$ & $17,56(3,75)$ & $5,49 * *$ \\
\hline Motivación superficial & $11,35(3,28)$ & $11,8(3,68)$ & $11,95(3,5)$ & $9,24(2,9)$ & $4,1^{* *}$ \\
\hline Estrategia superficial & $14,8(3,48)$ & $15,88(3,38)$ & $15,11(2,76)$ & $13,08(3,71)$ & $4,8^{* *}$ \\
\hline Enfoque profundo & $31,22(5,26)$ & $30,76(5,05)$ & $31,18(5,56)$ & $35,64(7,18)$ & $5,27 * *$ \\
\hline Enfoque superficial & $26,15(6,19)$ & $27,68(6,54)$ & $27,06(5,52)$ & $22,32(6,05)$ & $5,1 * *$ \\
\hline
\end{tabular}




\section{Discusión}

\section{Enfoques adoptados por los estudiantes}

Los resultados muestran que la mayoría de los participantes no se integran en ninguna de las categorías establecidas. Este grupo de alumnos y alumnas se suelen caracterizar por estar motivados intrínsecamente ante la tarea, disfrutando con el desarrollo de ésta y siendo su fin la comprensión. Éstos utilizan no sólo las estrategias típicas del enfoque profundo (que les posibilitan conocer el significado de la tarea, relacionar los contenidos con la experiencia y conocimientos previos); sino también estrategias superficiales (limitándose a lo esencial, centrándose sólo en los aspectos trabajados en clase y que serán materia de examen). En definitiva, buscan la comprensión del material de examen, sin preocuparse de ampliar el material por cuenta propia.

Uno de los presupuestos de la teoría SAL es la influencia que ejerce el contexto sobre los procesos de estudio del alumnado. Esta peculiaridad de los enfoques nos permite dar una explicación de porqué la mayoría del alumnado no se incluye en ninguna de las categorías. El alumnado posee unos motivos y utiliza unas estrategias que dependen en gran medida de las características del proceso de enseñanza (métodos de enseñanza y evaluación). Por tanto, estas características pueden provocar que el alumnado posea una mayor motivación profunda y utilicen estrategias superficiales en un alto grado. Posiblemente, esto se deba a que las consideran más adecuadas al contexto en el que aprenden. Un ejemplo de ello pueden ser las evaluaciones centradas en ofrecer un material específico para superar un examen. Estos métodos de evaluación pueden provocar que el alumnado esté interesado en comprender el material de examen y no necesite prestar atención al complementario.

Se han comparado los resultados de este estudio con los de otras investigaciones realizadas en contextos similares (por ejemplo, la misma universidad, titulación). Sin embargo, estos estudios tienen algunas diferencias con respecto a éste. Una de ellas es el cuestionario utilizado: Buendía y Olmedo (2003) utilizan el cuestionario ampliado (SPQ) y obtienen que el alumnado de la titulación de Pedagogía de la Universidad de Granada posee mayoritariamente un enfoque superficial, seguido de un enfoque profundo y finalmente de alto-rendimiento o logro. Otro estudio que utiliza el cuestionario ampliado (SPQ) es el realizado en la Universidad de Murcia. En éste se obtiene una media más alta del enfoque superficial en el alumnado 
de la titulación de Magisterio (Hernández Pina, García y Maquilón, 2001). A diferencia de estos estudios, los participantes de esta investigación adoptan un enfoque superficial o profundo en proporciones muy similares.

Son pocas las investigaciones destinadas a conocer cuáles son los enfoques de estudio que el alumnado adopta en la universidad. Además, los realizados no especifican los criterios utilizados para agrupar al alumnado en los diferentes enfoques. Sin embargo, de los resultados obtenidos en este estudio y considerando otros trabajos (Buendía y Olmedo, 2003; Hernández Pina y otros, 2001; Kember, 1996, 2000), podemos concluir varias ideas sobre los enfoques que adopta el alumnado universitario:

- Algunos estudios encuentran que un gran número de alumnos y alumnas no se pueden clasificar en un único enfoque y proponen que los enfoques de aprendizaje deberían estudiarse en un continuo. Los extremos de este continuo corresponden al enfoque superficial y al profundo. Los resultados de nuestro trabajo apoyan la necesidad de profundizar en el estudio de este continuo.

- La influencia del contexto puede causar que los enfoques adoptados mayoritariamente por los estudiantes de una misma titulación sean contradictorios. Esta influencia puede convertirse en un obstáculo para generalizar las características del aprendizaje del alumnado según las titulaciones u otros aspectos. Cabe decir que el interés del estudio de los enfoques de aprendizaje se centra más en la intervención para la mejora del proceso de enseñanza-aprendizaje de un contexto y unos estudiantes determinados, que en la generalización y obtención de perfiles de estudiantes según una titulación.

\section{Influencia del género y la edad del alumnado en sus enfoques de aprendizaje}

La investigación en la influencia del género en los enfoques de aprendizaje no es muy común en este ámbito de estudio, y en los casos en que se analizan, o no se encuentran diferencias (Hernández Pina y otros, 2002), o éstas sólo se dan en algunas de las escalas e ítems (Olmedo, 2001; Sadler-Smith y Tsang, 1998; Zhang, 2000). A diferencia de estos estudios, los resultados de esta investigación aportan diferencias en el género en todas las escalas y enfoques. 
El estudio de Cano (2000) refleja que las alumnas utilizan más el enfoque superficial y estratégico. Las diferencias encontradas en este trabajo son contradictorias con las encontradas en nuestro estudio. Nuestros resultados muestran que el enfoque profundo es significativamente más adoptado por las alumnas, siendo el superficial más adoptado por los alumnos. Esta contradicción puede deberse a que la muestra de ambos estudios pertenecen a tipos de carreras diferentes, siendo los participantes del estudio de Cano (2000) estudiantes de carreras de ciencias.

Las variables contextuales parecen influir en los enfoques de aprendizaje. En el caso de las diferencias de género puede decirse que estas influencias contextuales provienen de dos momentos diferentes. De un lado, las influencias contextuales referidas a la experiencia que hombres y mujeres han vivido dependiendo del sexo, debidas a un momento socio-cultural. De otro lado, la influencia que pueden ejercer las características del proceso de enseñanzaaprendizaje. Tanto unas influencias como otras han sido poco estudiadas; sin embargo, el estudio de la interrelación de estas variables se presenta como una interesante prospectiva de esta investigación.

A pesar de que la edad ha sido poco investigada, los resultados obtenidos en este trabajo confirman los obtenidos por otros autores (Hernández Pina y otros, 2002; Richardson, 1995; Sadler-Smith, 1996). Se obtiene que el alumnado de mayor edad adopta más el enfoque profundo. El alumnado con mayor edad posee más años de experiencia como aprendices, dicha experiencia es una de las características a considerar en los aprendices expertos (Justicia, 2000). Aunque se necesitan otras condiciones para llegar a ser aprendices expertos y con ello un mayor grado de comprensión en su aprendizaje, esta experiencia puede ser una de las razones por las que el alumnado de mayor edad adopta el enfoque profundo.

Concluyendo, la teoría de la SAL defiende que los enfoques de aprendizaje de los estudiantes dependen más de variables contextuales que individuales; sin embargo, no se excluye la influencia de éstas últimas (Biggs, 1993, 2001a, 2001b). En este sentido, los resultados de ésta y otras investigaciones (Sadler-Smith y Tsang, 1998; Zhang, 2000) muestran diferencias significativas según las características personales. Al igual que otros autores, sugerimos el aumento de estudios destinados a conocer las influencias de las diferencias individuales del alumnado, así como el porqué de estas diferencias. 
Para finalizar, alguna de las limitaciones de este estudio se centra en el método de muestreo utilizado, ya que no permite que estos resultados y conclusiones pueden ser generalizados al resto del alumnado.

\section{Prospectivas de futuro}

A partir de los resultados de nuestro estudio, pueden ofrecerse algunas de las conclusiones que debemos considerar en los futuros cambios de la enseñanza superior. Principalmente, puede producirse un desajuste entre los métodos de enseñanza-aprendizaje propuestos en los Créditos ECTS y las características señaladas de los procesos de estudio del alumnado. En este sentido, dichas características pueden propiciar el fracaso de métodos como la tutorización. Por tanto, nuestra propuesta gira en torno a la realizada por Vermunt (2003), en la que se manifiesta la necesidad de formar a un alumnado más autónomo en su aprendizaje, comenzando desde la enseñanza secundaria. Sin embargo, ante los nuevos cambios, el profesorado universitario debiera considerar la necesidad de cambiar paulatinamente las características del proceso de enseñanza para modificar los procesos que el alumnado acostumbra a adoptar en el sistema actual, y propiciar en éste la autorregulación del aprendizaje a través de la regulación de la enseñanza (De la Fuente et al, 2003-2006; De la Fuente y Justicia, 2004; De la Fuente, Justicia y Berbén, en prensa).

\section{Referencias}

Barca, A., Porto, A. y Santorum, R. (1997). Los enfoques de aprendizaje en contextos y situaciones educativas, una aproximación conceptual y metodológica. En A. Barca, J.L. Marcos, J.C. Núñez, A.M. Porto y M.R. Santorum (Eds.), Procesos de aprendizaje en ambientes educativos (pp. 387-435). Madrid: Centro de Estudios Ramón Areces.

Biggs, J. (1993). What do inventories of students' learning processes really measure? A theoretical review and clarification, British Journal of Educational Psychology, 63, 3-19.

Biggs, J. (2001a). Teaching for Quality Learning at University (3 ${ }^{\mathrm{a}}$ Ed.). Buckingham: Open University Press.

Biggs, J. (2001b). Enhancing learning: a matter of style or approach? En R.J. Sternberg y L.F. Zhang, Perspectives on Thinking, Learning and Cognitive Style (pp. 73-102). London: LEA.

Biggs, J., Kember, D. y Leung, D. (2001). The revised two-factor Study Process Questionnaire: R-SPQ-2F. British Journal of Educational Psychology, 71, 133-149. 
Buendía, L. y Olmedo, E. (2000). Estrategias de aprendizaje y procesos de evaluación en la educación universitaria. Bordón, 52 (2), 151-163.

Buendía, L. y Olmedo, E. (2002). El género: ¿constructo mediador en los enfoques de aprendizaje universitario? Revista de Investigación Educativa, 20 (2), 511-524.

Buendía, L. y Olmedo, E. (2003). Estudio transcultural de los enfoques de aprendizaje en educación superior. Revista de Investigación Educativa, 21 (2), 371-386.

Cano, F. (2000). Diferencias de género en estrategias y estilos de aprendizaje. Psicothema, 12 (3), 360-367.

Cano, F. y Justicia, F. (1994). Learning strategies, styles and approaches: an analysis of their interrelationships. Higher Education, 27, 239-260.

De la Fuente, J., Justicia, F., Cano, F., Pichardo, M.C., Martínez, J.M. y Berbén, A.B. (20032006). Mejora de la autorregulación del aprendizaje, en estudiantes universitarios, a través de estrategias de enseñanza reguladora "on-line". Proyecto $\mathrm{I}+\mathrm{D}+\mathrm{i}$ ref. BSO2003-06493. Madrid: Ministerio de Ciencia y Tecnología.

De la Fuente, J. y Justicia, F. (2004). Regulación de la enseñanza para la autorregulación del aprendizaje en la Universidad. Aula Abierta, 82, 161.

De Fuente, J., Justicia, F. y Berbén, A.B. G. (en prensa). El aprendizaje experto y la autorregulación del aprendizaje. En F. Justicia, M.C. Pichardo y E. Fernández, Enciclopedia de Psicología Evolutiva y de la Educación. Málaga: Aljibe.

De la Fuente, J. y Martínez, J.M. (2003). Cuestionario revisado del proceso de estudio. Versión castellana. Universidad de Almería. No publicado.

De la Fuente, J., Justicia, F., Archilla, I. y Soto, A. (1994). Factores condicionantes de las estrategias de aprendizaje y del rendimiento en alumnos universitarios a través del $A C R A$. Almería: Universidad de Almería.

Entwistle, N.J. y Ramsden, P. (1983). Understanding student learning. London: Croom Helm.

Entwistle, N.J. y Tait, H. (1990). Approaches to learning, evaluations of teaching, and preferences for contrasting academic environments. Higher Education, 19, 169-194.

Fox, R., McMacnus, I.C. y Winder, B. (2001). The shortened Study Process Questionnaire: An investigation of its structure and longitudinal stability using confirmatory factor analysis. British Journal of Educational Psychology, 71, 511-530. 
García, A., Salvador, L. y Zubieta, J.C. (1991). Elementos para un análisis evaluativo de la Universidad. El caso de la Universidad de Cantabria. Actas de las Jornadas "la investigación educativa sobre la universidad”. Madrid: CIDE.

Harris, J.M., Wickline, V.B. e Iliescu, C.S. (2004). Construct Validation of Revised Study Process Questionnaire (R-SPQ-2F) with an American College Sample. Poster presentado al $16^{\text {th }}$ Annual Convention. Chicago, Illinois, del 27 al 30 de mayo. http://www.psychologicalscience.org/convention/program/search/results.cfm?AbAuth or=40184 (Consultado julio 2004).

Hernández Pina, F. (1993). Concepciones en el estudio del aprendizaje de los estudiantes universitarios, Revista de Investigación Educativa, 22, 117-150.

Hernández Pina, F. (1997) El aprendizaje de los alumnos en el marco del Plan Nacional de Evaluación de las Instituciones Universitarias. En H. Salmerón (Ed.), Evaluación Educativa (pp. 175-206). Granada: GEU

Hernández Pina, F., García, M.P. y Maquilón, J.J. (2001). Estudio empírico de los enfoques de aprendizaje de los estudiantes universitarios en función del perfil de su titulación (profundo vs. superficial). Revista de Orientación y Psicopedagogía, 12 (22), 303318.

Hernández Pina, F., García, M.P., Martínez, P., Hervás, R.S. y Maquilón, J. (2002). Consistencia entre motivos y estrategias de aprendizaje en estudiantes universitarios. Revista de Investigación Educativa, 20 (2), 487-510.

Justicia, F. (2000). El comportamiento experto. En D. González, J.A. Amezcua y F. Peñafiel (Coords). El psicopedagogo en la organización y gestión de programas de formación (pp. 89-103). Granada: GEU.

Kember, D. (1996). The intention to both memorise and understand: another approach to learning? Higher Education, 31, 341-351.

Kember, D. (2000). Action learning and action research: improving the quality of teaching and learning. London: Kogan Page.

Kember, D. y Gow, L. (1994). Orientations to teaching and their effect on the quality of student learning. Journal of Higher Education. 65, 59-74.

Leung, M. y Chan, K. (2001).Construct Validity and Psychometric Properties of the Revised Two-factor Study Process Questionnaire (R-SPQ-2F) in the Hong Kong Context. Paper presented at the AARE 201 Conference, 2-6 December, 2001 at the Notre Dame University, Perth, Australia. 
Martín, F. y Camarero, F. (2001). Diferencias de género en los procesos de aprendizaje en universitarios. Psicothema, 13 (4), 598-604.

Marton, F. (1981). Phenomenography-describing conceptions of the world around us. Institutional Science, 10, 177-200.

Meyer, J.H., Dune, T.T. y Richardson, J.T.E. (1994). A gender comparison of contextualized study behaviour in higher education. Higher Education, 27, 469-485.

Olmedo, E.M. (2001). Estrategias de aprendizaje y modelos de enseñanza en educación superior. Tesis doctoral defendida en la Universidad de Granada.

Pilcher, R. (2002). Student Approaches to Learning in Accounting. Working Paper.3/02. http://www.csu.edu.au/faculty/commerce/research/research/2002/03-02.htm. (Consultado octubre 2003)

Richardson, J.T.E. (1995). Mature students in higher education: II. An investigation of approaches to studying and academic performance. Studies in Higher Education, 20, 517.

Sadler-Smith, E. (1996). Approaches to studying: age, gender and academic performance. Educational Studies, 22, 367-379.

Sadler-Smith, E. y Tsang, F. (1998). A comparative study of approaches to studying in Hong Kong and the United Kingdom. British Journal of Educational Psychology, 68, 81-93.

Trigwell, K., Prosser, M. y Waterhouse, F. (1999). Relations between teachers' approaches to teaching and students' approaches to learning. Higher Education, 37, pp.57-77.

Vermunt, J.D. (2003). The power of learning environments and the quality of student learning. En E. De Corte, L. Verschaffel, N.Entwistle y J. van Merriënboer (Eds.), Powerful Learning Environments: Unravelling Basic Components and Dimensions. London: Pergamon y Earli.

Zhang, L. (2000). University students' learning approaches in three cultures: an investigation of Biggs's 3P Model. The Journal of Psychology, 134 (1), 37-55. 